\title{
Deducción de Pérdidas de Potencia por Conducción en Inversores Modulación Senoidal de Ancho de Pulso, SPWM
}

\author{
Juan D. Gallego-Gomez, Juan B. Cano-Quintero y Nicolas Muñoz-Galeano \\ Fac. de Ingeniería, Depto Ing. Eléctrica, Grupo de Manejo Eficiente de la Energía - GIMEL, Universidad de \\ Antioquia, Calle 67 No. 53-108, Oficina 19-437, Medellín-Colombia (e-mail: jdgallego87@yahoo.es, \\ jbcano@gmail.com, nicolas.munoz@udea.edu.co)
}

Recibido Jul. 31, 2014; Aceptado Oct. 20, 2014; Versión final recibida Ene. 7, 2015

\begin{abstract}
Resumen
Este artículo presenta la deducción matemática de las pérdidas de potencia por conducción en inversores monofásicos de puente H controlados mediante Modulación Senoidal de Ancho de Pulso (SPWM). Se presenta el desarrollo matemático de las ecuaciones de voltaje y corriente a través de los semiconductores durante su estado de conducción y se calcula su disipación de potencia. Las ecuaciones de pérdidas de potencia encontradas dependen de las características de la modulación empleada y de los parámetros de la carga. Mediante validación experimental y de simulaciones se obtuvieron curvas que muestran la validez de las ecuaciones y sus aproximaciones.
\end{abstract}

Palabras clave: pérdidas de potencia por conducción, semiconductores, modulación SPWM, inversor de potencia

\section{Deduction of Power Losses by Conduction in Sinusoidal Pulse Width Modulation Inverters, SPWM}

\begin{abstract}
This paper presents the mathematical derivation of the power losses by conduction in single phase $\mathrm{H}$-bridge inverters controlled by Sinusoidal Pulse Width Modulation (SPWM). The development of the mathematical equations for voltage and current through the semiconductor in its conducting state is presented and the power dissipation is calculated. The power loss equations found depend on the characteristics of the modulation used and on the parameters of the load. Through simulations and experimental validation, curves showing the validity of the equations and their approaches in accordance with the conditions of the test were obtained.
\end{abstract}

Keywords: power losses by conduction, semiconductors, SPWM modulation, power inverter 


\section{INTRODUCCIÓN}

Los inversores de potencia de puente $\mathrm{H}$ sirven para transformar el voltaje, la frecuencia, la potencia, y el número de fases de acuerdo a los requerimientos de las cargas eléctricas. Están compuestos por interruptores de potencia controlados mediante técnicas de modulación. Las técnicas de modulación más usadas son la modulación senoidal de ancho de pulso (Senoidal Pulse Width Modulation, SPWM) y la modulación de espacio vectorial (Space Vector Modulation, SVM) Rus et al. (2010), aunque existen múltiples modulaciones derivadas de las modulaciones SPWM y SVM. Las principales aplicaciones de los inversores de potencia de puente $\mathrm{H}$ son: variadores de frecuencia Kojabadi (2011), compensadores activos Orts et al. (2010), sistemas de alimentación ininterrumpida Komurcugil (2012), convertidores de una fase a tres fases Kim et al. (2002) y Jacobina et al. (2010), entre otras.

La eficiencia y la potencia disipada en los inversores de potencia son parámetros de diseño primordiales para la determinación de los sistemas de disipación de calor y del consumo de energía en general en los inversores. En este sentido, es necesario un modelo matemático que permita calcular las pérdidas de potencia. Las pérdidas de potencia en inversores se dividen en las perdidas dinámicas debidas a la conmutación de los interruptores y en las perdidas por conducción. Las pérdidas por conmutación dependen principalmente de la frecuencia de conmutación y de características físicas de los semiconductores. Mientras que las pérdidas por conducción dependen de la carga del inversor y de las caídas de voltaje en régimen estático en los semiconductores de los interruptores de potencia. En el presente artículo se presentarán las ecuaciones que modelan las pérdidas de potencia por conducción en semiconductores para la modulación SPWM presentada en Gallego et al. (2014) en inversores monofásicos. Las pérdidas por conducción para otras modulaciones pueden deducirse fácilmente a partir del procedimiento utilizado en este artículo. Los resultados pueden extrapolarse fácilmente a convertidores trifásicos.

Existen diferentes maneras de implementar la modulación SPWM para inversores monofásicos en configuración puente H. Usando cuatro señales digitales características de la modulación unipolar(Xiao et al., 2011). También se puede generar una señal SPWM bipolar en la carga mediante dos señales digitales Wang et al. (2012). De acuerdo con la forma en que se generan las señales digitales a través de dispositivos electrónicos de control, se puede generar una modulación SPWM a través de FPGAs M. N. Md Isa (2007) o usando un microcontrolador Ismail et al. (2006). Para los estudios de este artículo, se usara la modulación SPWM reportada en Gallego et al. (2014), la cual está basada en el uso de cuatro interruptores cuyas señales digitales se obtienen al comparar una señal triangular con una señal senoidal.

Las pérdidas de potencia por conducción en inversores SPWM han sido estudiadas en detalle en distintas obras, a continuación se mencionan las más representativas: Xiao et al. (2011) realiza una comparación de pérdidas de potencia para varias topologías moduladas mediante SPWM unipolar. En Perantzakis et al. (2007) se propone una nueva topología de convertidor con referencia a un NPC de cuatro niveles basado en la distribución de pérdidas de potencia por conducción y por conmutación. Bazzi et al. (2012) realiza la estimación de las pérdidas por conducción mediante la medida de las variables de voltaje y corriente en los conductores. También se han usado modelos basados en funciones de transferencia y funciones de conmutación para el cálculo de las perdidas por conducción Hosseini Aghdam et al. (2005). El cálculo de las perdidas por conducción en inversores modulados mediante SPWM ha sido estudiado mediante métodos analíticos en Casanellas (1994), Dahono et al., (1995), Bierhoff et al. (2004), algunas ecuaciones presentadas en estos artículos se tomaron como base para la deducción del nuevo modelo. A partir de los parámetros del inversor, de los parámetros de la carga, y las características de la modulación se proponen expresiones matemáticas para elmodelo que pueden ser corroboradas a través de simulaciones y experimentos. Las expresiones deducidas a través de la deducción teórica no han sido reportadas hasta ahora en la literatura técnica. Su conocimiento permite ampliar la construcción de herramientas teóricas para el estudio energético de los inversores controlados mediante modulaciones SPWM, sus variantes y modulaciones similares.

La deducción de las pérdidas de potencia por conducción se lleva a cabo mediante la deducción de ecuaciones de corriente y voltaje a través de los semiconductores. Estas ecuaciones se determinan a partir de las formas de onda típicas propias de la modulación. En la determinación de las ecuaciones debe incluirse el ciclo de servicio, los tiempos de conducción y el índice de modulación. Obtenidas las ecuaciones de corriente y voltaje se suman las perdidas energéticas para hallar la potencia promedio en cada semiconductor. Los resultados teóricos se verifican por simulaciones a través de MATLAB-simulink. También se verifican mediante experimentos midiendo el voltaje y la corriente a través de los semiconductores. Los métodos de análisis para la obtención de las pérdidas de potencia pueden extenderse a otro tipo de inversores SPWM. 


\section{INVERSOR DE POTENCIA Y MODULACIÓN SPWM}

El inversor monofásico de la figura $1 \mathrm{~b}$ está compuesto por un bus $\mathrm{DC}$, un puente $\mathrm{H}$ de dos ramas, y una carga AC. Cada rama contiene dos interruptores que conmutan de acuerdo a la estrategia de modulación. Cada interruptor (11, I2, I3 e 14) está compuesto por un IGBT (Q1, Q2, Q3 y Q4) y un diodo en antiparalelo (D1, D2, D3 y D4). El disparo de los interruptores se realiza mediante señales digitales enlas puertas G1, G2, G3, y G4. El inversor convierte la tensión DC del bus de continua en tensión alterna AC inyectando las corrientes que requiere la carga.

Los interruptores tienen tres posibles estados de conducción. En estado de conducción de los IGBT (Q) la corriente que atraviesa el IGBT del interruptor es lo y el voltaje colector emisor $v_{C E}$ es positivo. En estado de conducción del diodo (D) la corriente por el diodo del interruptor es lo y el voltaje colector emisor $v_{C E}$ es negativo. $Y$ en el estado en que ninguno conduzca el interruptor es un circuito abierto y el voltaje colector emisor es Vs. Las dos primeras condiciones de conducción se muestran en la figura 1a.

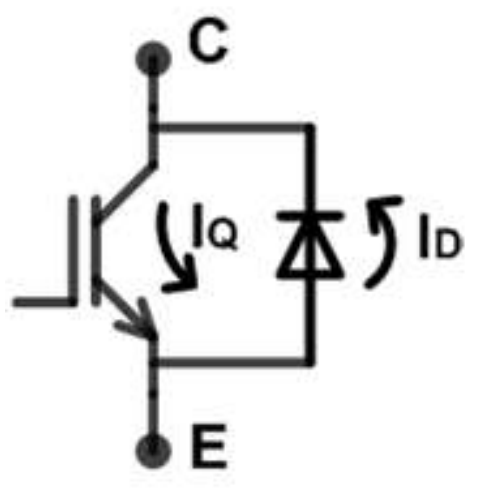

a)

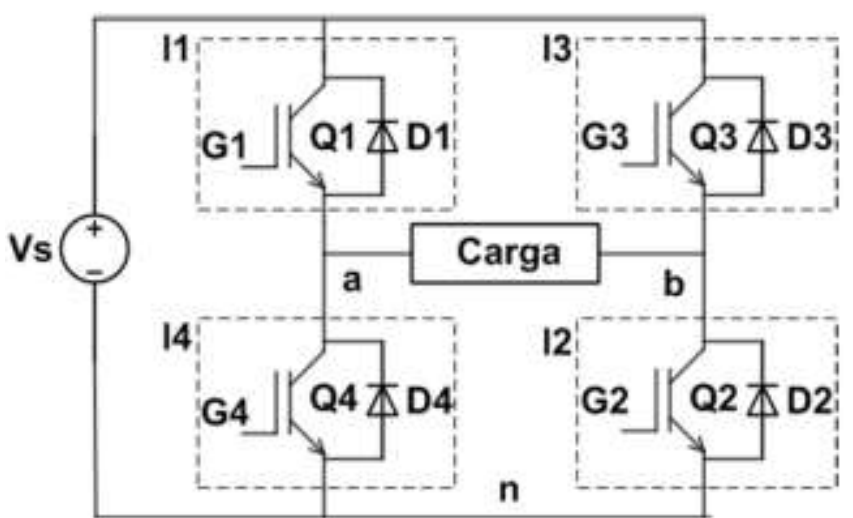

b)

Fig.1: Inversor topología puente $\mathrm{H}$ y corrientes en los interruptores.

Existen diferentes tipos de modulación para controlar el disparo de los interruptores, en este artículo se utiliza la estrategia de modulación SPWM porque es una de las modulaciones más usada en los inversores de potencia comerciales. La modulación SPWM consiste en comparar una señal de referencia (senoidal) con una señal portadora (triangular), ver figura 2. La comparación genera un tren de pulsos con ancho variable, a continuación se describen como se generan las señales de control (disparo) para G1, G2, G3, y G4.

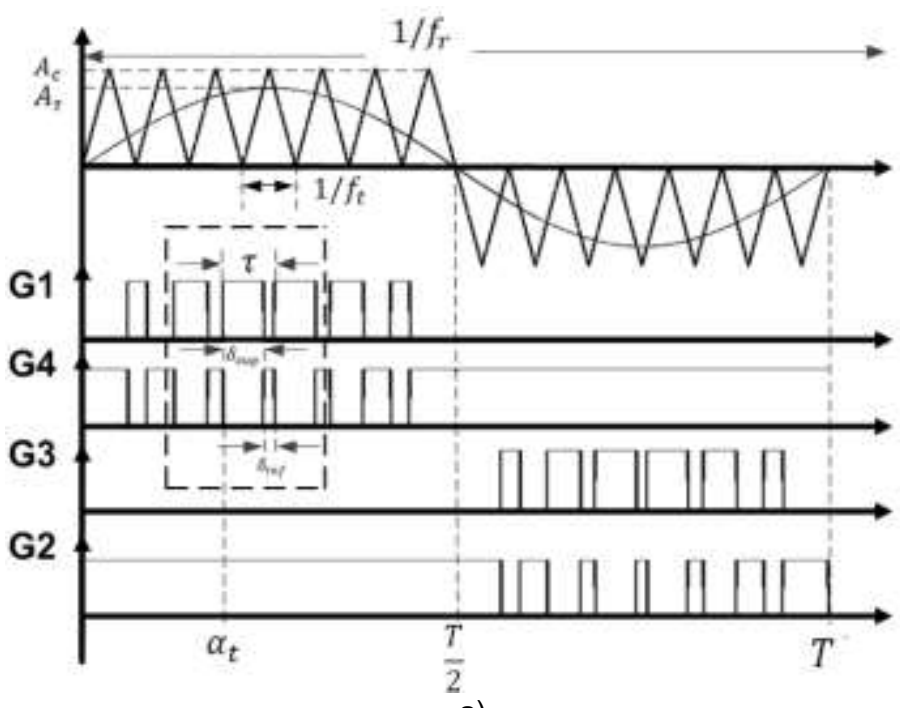

a)

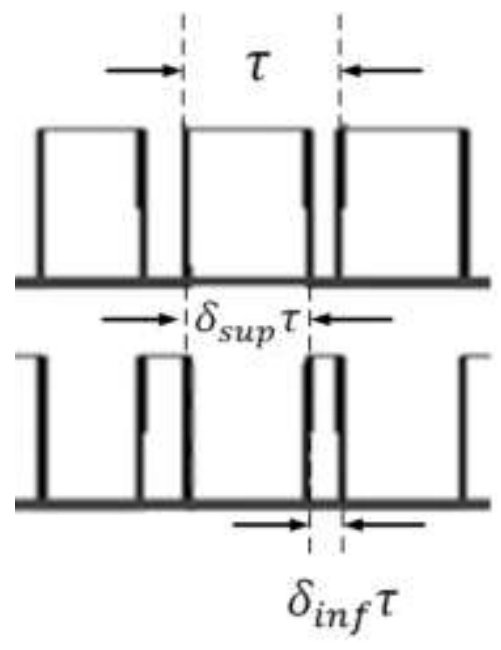

b) zoom recuadro.

Fig.2: Generación de los pulsos para la modulación SPWM. 
1) Semiciclo positivo para las señales de referencia y portadora: la señal de control G1 se obtiene a partir de la comparación de la señal de referencia con la portadora, tiene valor digital alto cuando la señal senoidal es mayor que la señal triangular. La señal G4 es el complemento digital de G1. La señal G3 tiene valor digital bajo y la señal G2 tiene valor digital alto por ser su complemento.

2) Semiciclo negativo para las señales de referencia y portadora: la señal de control G3 se obtiene a partir de la comparación de la señal de referencia con la portadora, tiene valor digital alto cuando el valor absoluto de la señal senoidal es mayor que el valor absoluto de la señal triangular, mientras que la señal G2 es el complemento de G3. La señal de control G1 tiene valor digital bajo y G4 tiene valor digital alto.

Esta modulación permite convertir la tensión $\mathrm{DC}(\mathrm{Vs})$ en tensión alterna entre los bornes a y b de la figura 1. El análisis de las pérdidas de potencia en los semiconductores del inversor depende de las señales de disparo (G1, G2, G3, y G4). La siguiente sección presenta la deducción de las pérdidas por conducción del inversor con base en la señales de disparo del inversor.

\section{PERDIDAS DE POTENCIA POR CONDUCCIÓN EN INVERSORES SPWM}

En la presente sección se deducen las pérdidas de potencia asociadas a la conducción en cada uno de los dispositivos semiconductores del inversor cuando se implementa la modulación SPWM cuyas señales de referencia y portadora están digitalizadas Trigg et al. (2008). Las expresiones obtenidas dependen de las características de la modulación utilizada, de las magnitudes de voltaje y de las corrientes que atraviesan el dispositivo en cuestión. Para el análisis se considera una carga resistiva-inductiva (RL).

\section{Deducción de tiempos de conducción y ciclos de servicio}

Para hallar las pérdidas de potencia en cada semiconductor deben definirse las cantidades acotadas de la figura $2\left(\delta_{\text {sup }}, \delta_{\text {inf }}, \tau\right.$ y $\left.\alpha_{t}\right)$. Estas cantidades representan las características geométricas de la modulación y las expresiones de las pérdidas de potencia deben incluirlas. Al tomar un pulso cualquiera del tren, $\alpha_{t}$ es la posición en el eje del tiempo de ese pulso (flanco de subida). El período de conmutación $\tau$ es el tiempo que hay entre pulsos. El producto $\delta_{\text {sup }} \tau$ se define como el tiempo durante el cual los interruptores superiores (I1 o I3) tienen señal de puerta, $\delta_{\text {sup }}$ suele representarse como una fracción de $\tau$ mediante (1)Trigg et al.(2008).

$\delta_{\text {sup }}\left(\alpha_{t}\right)=M * \operatorname{sen}\left(\omega_{1} \alpha_{t}\right)$

Donde $M$ es el índice de modulación en amplitud dado por $M=A_{c} / A_{r}$ (donde $A_{c}$ es la amplitud de la portadora y $A_{r}$ es la amplitud de la referencia, figura 2) y $\omega_{1}$ es la frecuencia angular fundamental $\left(\omega_{1}=2 \pi / T\right)$. Mientras que el tiempo durante el cual los interruptores inferiores (I2 e 14) tienen señal de puerta $\delta_{\text {inf }} \tau$ es el tiempo restante del periodo $\tau$. La fracción $\delta_{\text {inf }}$ está dada por (2):

$\delta_{\text {inf }}\left(\alpha_{t}\right)=1-M * \operatorname{sen}\left(\omega_{1} \alpha_{t}\right)$

\section{Expresión general para la determinación de potencias}

La energía disipada por cualquier interruptor del puente durante la conducción de corriente depende de la caída de voltaje en estado de conducción de la corriente, del ancho del pulso $\delta$ (que puede ser $\delta_{\text {sup }}$ para los interruptores superiores o $\delta_{i n f}$ para los interruptores inferiores) y del periodo de conmutación. Todas estas cantidades evaluadas en la coordenada $\alpha_{t}$ dada en segundos. La expresión para la energía en cada pulso está dada por (3)Casanellas (1994).

$E_{\text {loss }}=v_{C E}\left(\alpha_{t}\right) * i_{c}\left(\alpha_{t}\right) * \delta\left(\alpha_{t}\right) * \tau$

Donde $v_{C E}\left(\alpha_{t}\right)$ es el voltaje instantáneo colector-emisor en estado de conducción del transistor. $i_{c}\left(\alpha_{t}\right)$ es la corriente instantánea que atraviesa el interruptor. El producto entre $\delta\left(\alpha_{t}\right)$ y $\tau$ es el tiempo en segundos que dura el pulso y sobre el cual se calcula la energía $E_{\text {loss. }}$. Para obtener la energía durante algún periodo referente se parte del diferencial de energía de la ecuación (4)Casanellas (1994).

$d E_{\text {loss }}=v_{C E}\left(\alpha_{t}\right) * i_{c}\left(\alpha_{t}\right) * \delta\left(\alpha_{t}\right) * d \tau$ 
Es conveniente expresar los términos de la ecuación (4) en coordenadas angulares (radianes) para obtener las pérdidas en un periodo completo de la señal alterna donde se repite la modulación. De acuerdo a lo anterior, se define la proporcionalidad de la ecuación (5).

$d \tau=\frac{d \alpha_{r} * T}{2 \pi}$

Donde $T$ es el periodo de la señal de referencia (figura 2). Reemplazando (5) en (4) se obtiene la energía infinitesimal cuyas coordenadas están en radianes:

$d E_{\text {loss }}=v_{C E}\left(\alpha_{r}\right) * i_{c}\left(\alpha_{r}\right) * \delta\left(\alpha_{r}\right) * \frac{d \alpha_{r} * T}{2 \pi}$

Al sumar las contribuciones de energía disipada durante cada pulso ( $\left.d E_{l o s s}\right)$ y dividirla luego entre el periodo $T$ se obtiene la potencia promedio en un período completo de tiempo:

$P_{\text {avg }}=\frac{1}{2 \pi} \int_{a}^{b} d E_{\text {loss }}=\frac{1}{2 \pi} \int_{a}^{b} v_{C E}\left(\alpha_{r}\right) * i_{c}\left(\alpha_{r}\right) * \delta\left(\alpha_{r}\right) * d \alpha_{r}$

Formas de onda de corriente y voltaje

Al conocer las funciones $v_{C E}\left(\alpha_{r}\right), i_{c}\left(\alpha_{r}\right), \delta\left(\alpha_{r}\right)$ y los límites $a$ y $b$ se pueden determinar de manera teórica las pérdidas por conducción en cada semiconductor del puente $\mathrm{H}$. La integral de la ecuación (7) puede separarse de acuerdo a los tiempos durante los cuales cada semiconductor del interruptor conduce y disipa energía.

En la figura 3 se muestra la forma general de las ondas de corriente para una carga resistiva-inductiva obtenida mediante análisis en Gallego et al.(2014). La conducción a través de los interruptores puede analizarse a partir del desfase $\theta$, el cual es el factor de potencia en la carga (dado por $\theta=\tan ^{-1} \frac{\left|\mathrm{j} \omega_{1} \mathrm{~L}\right|}{\mathrm{R}}$, con $\omega_{1}$ la frecuencia angular fundamental de la onda de tensión y L y R los parámetros de la carga).

De la figura 3 puede verse que la forma de onda de la corriente del interruptor 1 es igual a la forma de onda de la corriente del interruptor 3 (interruptores superiores), mientras que la forma de onda de la corriente del interruptor 2 es igual a la forma de onda de la corriente del interruptor 4 (interruptores inferiores), excepto que sus ondas están desfasadas por $\pi$ radianes. Es por esto que las pérdidas de potencia en los interruptores superiores son iguales (interruptores 1 y 3), y también son iguales las pérdidas en los interruptores inferiores (interruptores 2 y 4). Debido a lo anterior, sólo es necesario el análisis y la deducción de las ecuaciones para una sola rama (para el análisis se eligió la rama de interruptores 1 y 4 ).

Por convención, en la figura 3, la corriente cuando circula por los IGBTs es positiva, mientras que es negativa cuando circula por los diodos. Para simplificar el análisis se considerará que la caída de voltaje en los semiconductores $v_{C E}\left(\alpha_{r}\right)$ es constante. Para el cálculo de la potencia en los IGBT se usará $v_{C E}\left(\alpha_{r}\right)=V_{I G B T}$ y en los diodos $v_{C E}\left(\alpha_{r}\right)=-V_{D}$.

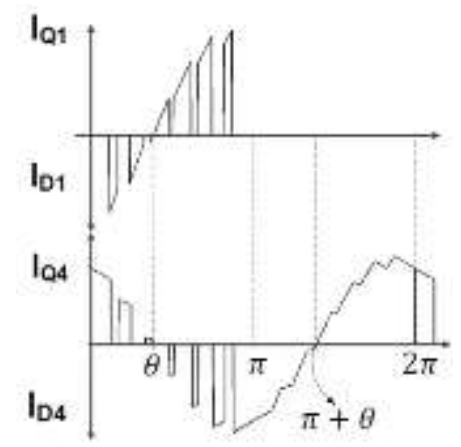

a. Interruptor 1 (superior), interuptor 4 (inferior)

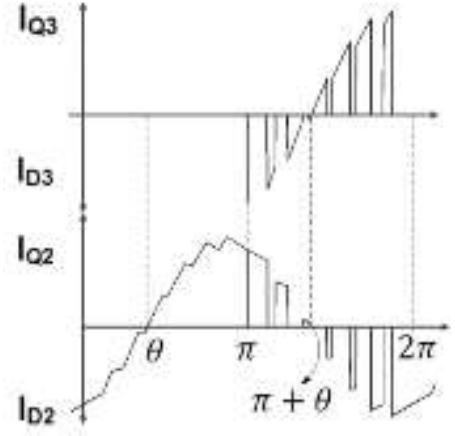

b. Interruptor 3 (superior), interuptor 2 (inferior)

Fig. 3: Formas de onda de corriente en los interruptores. 


\section{Formas concretas de las ecuaciones de potencia en cada semiconductor}

A continuación se deducirán las perdidas por conducción para cada semiconductor. Para ello se plantean las ecuaciones para $i_{c}\left(\alpha_{r}\right)$ para los interruptores superiores e inferiores. Para los dispositivos superiores puede modelarse de acuerdo a la ecuación (8) teniendo en cuenta el desfase $\theta$ con respecto al voltaje de la carga $\left(I_{c}\right.$ es el valor pico de la corriente de la carga). La figura 3a (inferior) muestra la forma de onda de la corriente del interruptor 4. Esta corriente tiene una fase de $\pi-\theta$ respecto al voltaje de salida en los bornes de salida ab del inversor de la figura 1, su expresión está dada por (9), por lo que esta ecuación modela la corriente de los dispositivos inferiores. En el caso de los diodos se usa la misma ecuación con signo negativo.

$I_{C_{-} \text {up }}(\alpha)=I_{c} * \operatorname{sen}\left(\alpha_{r}-\theta\right)$

$I_{C_{-} \text {down }}(\alpha)=I_{c} * \operatorname{sen}\left(\alpha_{r}+\pi-\theta\right)$

1) Pérdidas en los IGBTs superiores (Q1 yQ3): El IGBT Q1 conduce en el intervalo $(\theta, \pi)$ ya que tiene señal de puerta. Se utiliza $\delta_{\text {sup }}$ ya que se están calculando las pérdidas de los IGBTs superiores. Los límites entre los cuales Q1 conduce se pueden tomar directamente de la figura 3a, donde puede notarse que son entre $a=\theta$ y $b=\pi$. Reemplazando y solucionando (7), la potencia disipada en Q1 y Q3 $\left(P_{I Q 1}=P_{I Q 3}\right)$ se obtiene de ecn. (10)

$P_{I Q 1}=P_{I Q 3}=\frac{V_{I G B T} * I_{C} * M}{2 \pi}(\operatorname{sen}(\theta)+\pi * \cos (\theta)-\theta * \cos (\theta))$

2) Pérdidas en los IGBTs inferiores (Q2 y Q4): Se utiliza $\delta_{\text {inf }}$ ya que se están calculando las pérdidas de los IGBT's inferiores. El cálculo de la potencia debe hacerse mediante la suma de dos integrales: una con límites entre $a=0$ y $b=\theta$ y otra entre $a=\pi+\theta$ y $b=2 \pi$ donde conduce el IGBT4 (IQ4) (Ecuación 11). Esta última sin función de ancho de pulso involucrada ya que la señal de compuerta G4 tiene valor digital alto entre $\pi$ y $2 \pi$ (figura 2). Las dos integrales están expresadas en (11) y su solución en (12). Esta relación se cumple para los IGBT de las ramas inferiores por lo que representa también el cálculo de la potencia en Q2 $\left(P_{I Q 4}=P_{I Q 2}\right)$.

$$
\begin{aligned}
& P_{I Q 4}=\frac{1}{2 \pi}\left(\int_{0}^{\theta} V_{I G B T} * I_{Q 4}\left(\alpha_{r}\right) * \delta_{\text {inf }}\left(\alpha_{r}\right) * d \alpha_{r}+\int_{\pi+\theta}^{2 \pi} V_{I G B T} * I_{Q 4}\left(\alpha_{r}\right) * d \alpha_{r}\right) \\
& P_{I Q 4}=P_{I Q 2}=\frac{V_{I G B T} * I_{c}}{2 \pi}\left(2-M * \frac{\operatorname{sen}(\theta)}{2}+M * \theta * \frac{\cos (\theta)}{2}\right)
\end{aligned}
$$

3) Perdidas en los diodos superiores (D1 y D3): El diodo D1 conduce en el intervalo $(0, \theta)$ en el cual la corriente de carga es negativa. Se utiliza $\delta_{\text {sup }}$ ya que D1 solo conducirá si el interruptor Q4 se encuentra abierto. Los límites entre los cuales el diodo conduce se toman de la figura $3 a$, y puede notarse que son $a=0$ y $b=\theta$. Reemplazando y solucionando en (7) la potencia disipada en D1 y D3 $\left(P_{D 1}=P_{D 3}\right)$ se obtiene en la ecn. (13).

$P_{D 1}=P_{D 3}=\frac{V_{D} I_{C} M}{4 \pi}(\operatorname{sen}(\theta)-\theta * \cos (\theta))$

4) Perdidas en los diodos inferiores (D2 y D4): El diodo D4 conduce en los intervalos $(\theta, \pi)$ y $(\pi, \pi+\theta)$ en los cuales la corriente de carga es positiva. El cálculo de la potencia debe hacerse mediante la suma de dos integrales: una con límites entre $a=\theta$ y $b=\pi$ (intervalo en el cual la conducción en el diodo depende que el interruptor Q1 se encuentre abierto) y otra entre $a=\pi$ y $b=\pi+\theta$ (intervalo en el cual Q1 se encuentra permanentemente abierto y no hay función de ancho de pulso involucrada). El planteamiento de la integral se expresa en (14) y su solución en (15). Esta relación se cumple para los diodos de las ramas inferiores por lo que representa también el cálculo de la potencia en D2 $\left(P_{D 4}=P_{D 2}\right)$.

$$
\begin{gathered}
P_{D 4}=\frac{1}{2 \pi}\left(\int_{\theta}^{\pi} V_{D} * I_{Q 1}\left(\alpha_{r}\right) * \delta_{i n f}\left(\alpha_{r}\right) * d \alpha_{r}+\int_{\theta}^{\pi+\theta} V_{D} * I_{Q 1}\left(\alpha_{r}\right) * d \alpha_{r}\right) \\
P_{D 4}=P_{D 2}=\frac{V_{D} I_{c}}{2 \pi}\left(2-M * \frac{\operatorname{sen}(\theta)}{2}-M * \pi * \frac{\cos (\theta)}{2}-M * \theta * \frac{\cos (\theta)}{2}\right)
\end{gathered}
$$




\section{SIMPLIFICACION DE LAS ECUACIONES DE PERDIDAS DE POTENCIA}

En lo que sigue, se simplificarán las ecuaciones (10), (12) y (15) para obtener expresiones simples para las pérdidas de potencia en los semiconductores del inversor. Esto de acuerdo con las condiciones específicas de las pruebas experimentales y simulaciones. La corriente en la carga puede aproximarse a una corriente senoidal para valores altos del índice de modulación en frecuencia $m_{f}$. Esto permite analizar el valor de la corriente mediante la aproximación expresada en la ecuación (16) en la que se asume que la corriente sólo contiene la armónica fundamental. En este análisis también se considera el término $(\operatorname{sen}(\theta)-\theta * \cos (\theta))$ muy próximo a cero.

$I_{c}=\frac{\left|V_{1}\right|}{\left|\sqrt{\left(\omega_{1} \mathrm{~L}\right)^{2}+R^{2}}\right|}=\frac{M * V_{d c}}{\left|\sqrt{\left(\omega_{1} \mathrm{~L}\right)^{2}+R^{2}}\right|}$

1) Forma simplificada de la ecuación (10): Sustituyendo (16) en la ecuación (9) y teniendo en cuenta que el único parámetro que cambia es $M$ la potencia en Q1 puede aproximarse a la ecuación (17), con el parámetro $K_{1}=\frac{V_{I G B T} * V_{d c} \cos (\theta)}{2 * \sqrt{\left(\omega_{1} \mathrm{~L}\right)^{2}+R^{2}}}$ constante. En consecuencia la ecuación (9) se puede aproximar a una función cuadrática como la que se muestra en la figura 6a y la ecuación (17).

$P_{I Q 1}=K_{1} M^{2}$

2) Forma simplificada de la ecuación (12): En el caso de la ecuación (12), ésta se convierte en (19). En donde se han eliminado los dos últimos términos del segundo factor que tienen la forma $M^{2}(\operatorname{sen}(\theta)-\theta * \cos (\theta))$ que puede aproximarse a cero. El coeficiente que acompaña a la ecuación (18) $K_{2}=\frac{V_{I G B T^{*} V_{d c}}}{\pi * \sqrt{\left(\omega_{1} \mathrm{~L}\right)^{2}+R^{2}}}$ constante. Esto lleva a una relación lineal entre la potencia en Q4 y el índice de modulación como se muestra en la figura 6b.

$P_{I Q 4}=K_{2} M$

3) Forma simplificada de la ecuación (15): La sustitución de (16) en (15) lleva a la ecuación (19). Con los coeficientes $K_{3}=\frac{V_{D} V_{d c}}{\pi * \sqrt{\left(\omega_{1} \mathrm{~L}\right)^{2}+R^{2}}}$ y $K_{4}=\frac{V_{D} V_{d c}(\operatorname{sen}(\theta)+\pi * \cos (\theta)+\theta * \cos (\theta))}{4 * \pi * \sqrt{\left(\omega_{1} \mathrm{~L}\right)^{2}+R^{2}}}$ constantes. La ecuación (19) tiene la forma de una función parabólica cuya concavidad es negativa. Su gráfica se muestra en la figura 6c.

$P_{D 4}=K_{3} M-K_{4} M^{2}$

\section{DESCRIPCION DE LA SIMULACION}

Las ecuaciones (9), (12), (13) y (15) muestran la dependencia no lineal de las pérdidas en conducción de los semiconductores con respecto al factor de potencia y el índice de modulación. Con el fin de validar estas ecuaciones se han simulado en Matlab diferentes condiciones del inversor en puente $\mathrm{H}$. En la figura 4 se muestra el circuito de potencia que se ha construido con los bloques de simulink. Para generar las señales de la modulación SPWM (G1, G2, G3, G4) se utilizó la herramienta S-Function de Simulink. Una S-Function permite crear bloques en Simulink mediante código de matlab utilizando archivos de programación ".M".

Se considera la topología de la figura 1, en la cual se utilizan los siguientes valores para realizar la simulación: voltaje en el bus de continua $\left(V_{d c}=160 \mathrm{~V}\right)$, resistencia en la carga $\left(R_{\text {carga }}=21 \Omega\right)$, inductancia en la carga $\left(L_{\text {carga }}=45 \mathrm{mH}\right)$, frecuencia fundamental de salida del inversor $\left(f_{1}=60 \mathrm{~Hz}\right)$, el número de pulsos de la modulación $(n p=50)$ y la caída de tensión en IGBT y diodos $\left(V_{D}\right.$ y $\left.V_{I G B T}=1 \mathrm{~V}\right)$. El índice de modulación en frecuencia equivale a $m_{f}=2 *(n p+1)=f_{t} / f_{r}=102$. El índice de modulación $M$ varía desde 0.2 hasta 1 . Los valores ingresados en la simulación corresponden a los valores medidos en las pruebas experimentales. 


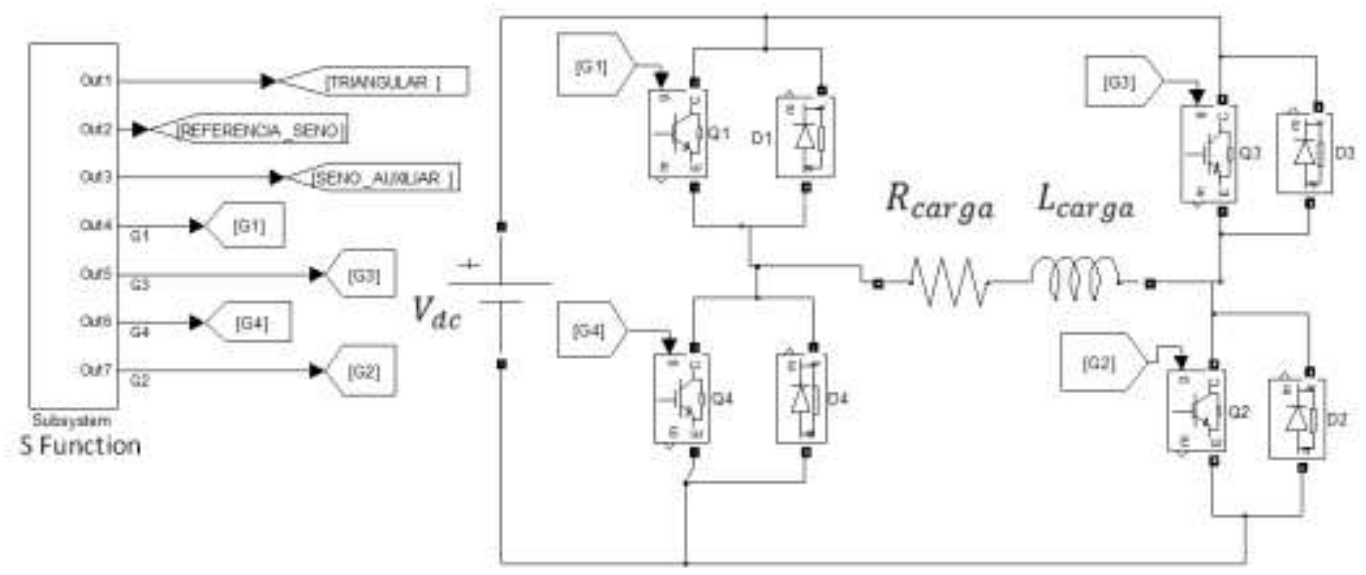

Fig.4: Simulación topología puente H Matlab-Simulink.

\section{DESCRIPCION DE LAS PRUEBAS EXPERIMENTALES}

La validación experimental se llevó a cabo mediante el montaje del hardware mostrado en la figura 5. Allí se muestra el montaje del puente $\mathrm{H}$ monofásico para el cual se usaron IGBTs de referencia IRG4PC40FD de International Rectifier. Las señales de disparo de los IGBTs (G1, G2, G3 y G4) generadas por el microcontrolador dsPIC30F4012 perteneciente a la familia Microchip se acondicionan a través de un circuito de gate drivers con integrados ISO5500 de Texas Instuments. Para la medida se usaron pinzas amperimétricas de marca Tektronix A622, y de voltaje de la marca Teledyne Lecroy AP031, un osciloscopio de dos canales de la marca Tektronix TDS2022B y un computador para procesar la información como se muestra en la figura 5.

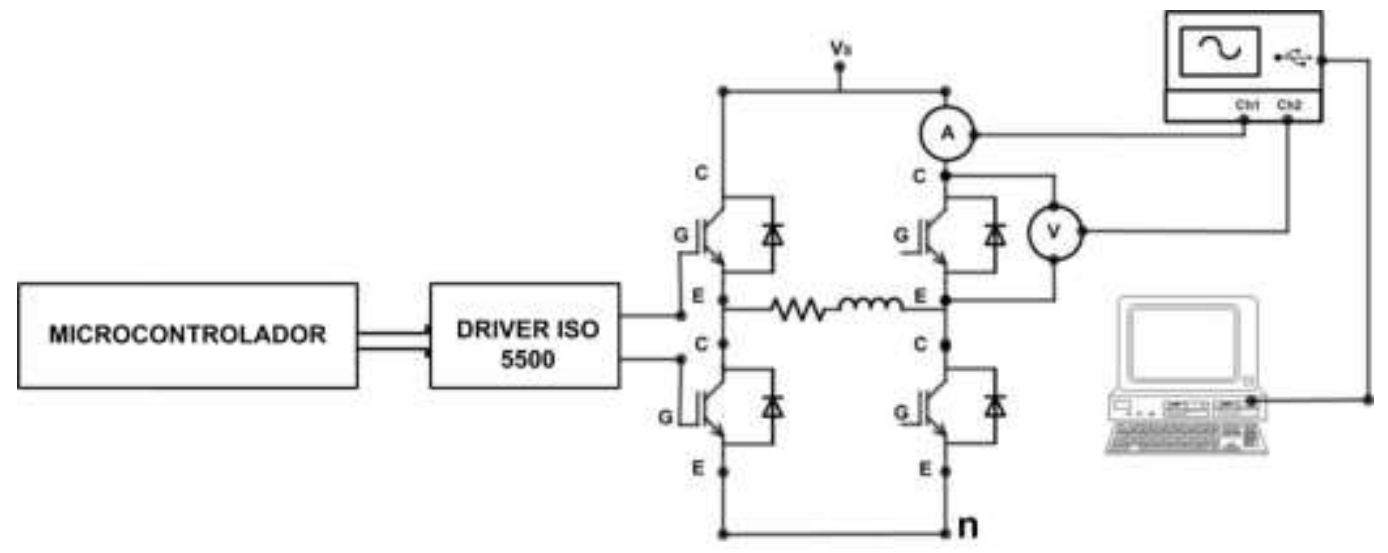

Fig. 5: Esquema de montaje para validación experimental.

La programación del microcontrolador se llevó a cabo con el software MPLAB en programación C. Desde allí, mediante matrices y vectores, se han programado las salidas PWM correspondientes a la modulación senoidal. El código para generar la modulación se desarrolló con los mismos parámetros indicados en la sección de descripción de la simulación. De igual manera las magnitudes de voltaje del bus DC y de los parámetros de la carga son los mismos que se usaron en las simulaciones.

La medición de las pérdidas de potencia se ha hecho de manera indirecta obteniendo la corriente y el voltaje a través de cada interruptor. El osciloscopio envía vectores que corresponden con la medida de voltaje y corriente (V y A en la figura 5) al computador. El computador -a través de MATLAB- efectúa el cálculo de la potencia disipada en cada dispositivo. Para el cálculo discreto de la potencia $\left(P_{d}\right)$ se utiliza la ecuación (20) Xiao et al. (2002), la cual es equivalente a la ecuación teórica (7).

$P_{d}=\frac{1}{N} \sum_{n=0}^{N-1} v\left(t_{i}\right) i\left(t_{i}\right)$ 
Donde $N$ es el número de muestras tomada por periodo de la fundamental y que proporciona el osciloscopio. $v\left(t_{i}\right)$ y $i\left(t_{i}\right)$ son cada uno de los elementos de los vectores de voltaje y corriente obtenidos en la medida. Para cada interruptor se diferencian las perdidas debidas al IGBT y al diodo, dependiendo del sentido de la corriente (figura 1a).

\section{COMPARACION DE RESULTADOS: CALCULADOS, SIMULADOS Y MEDIDOS}

En esta sección se hace la comparación de los resultados obtenidos mediante los cálculos con las ecuaciones propuestas, las simulaciones en matlab-simulink, y las pruebas experimentales en los interruptores de la rama izquierda del inversor (I1 e 14).Los parámetros de entrada para realizar los cálculos teóricos y las simulaciones fueron obtenidos a partir de la medición en el montaje experimental: el ángulo del factor de potencia dado por $\theta=\tan ^{-1} \frac{\left|\mathrm{j} \omega_{1} \mathrm{~L}\right|}{\mathrm{R}}=0.679 \mathrm{rad}$ con $\omega_{1}=2 \pi f_{1}=2 \pi 60$ y con $\mathrm{L}=45 \mathrm{mH}$ y $\mathrm{R}=21 \Omega$ y la caída de tensión en IGBT y diodos $\left(V_{D}\right.$ y $\left.V_{I G B T}=1 V\right), \mathrm{m}_{f}=102$.

El índice de modulación $M$ varía desde 0.2 hasta 1 . En la tabla 1 se muestran las pérdidas de potencia en función del índice de modulación $M$. Los resultados de la tabla 1 se grafican en la figura 6 para $P_{I Q 1}, P_{I Q 4}$ y $P_{D 4}$. La curva correspondiente a $P_{D 1}$ no se ha graficado debido a que sus valores son muy cercanos a cero. En la figura 6 , la línea continua corresponde con las potencias calculadas, la línea punteada corresponde con las potencias simuladas y la línea con guiones corresponde a las potencias que se midieron en las pruebas experimentales. En la tabla 1 también mostramos el error porcentual de las medidas en las pérdidas del IGBT4 $\left(\boldsymbol{P}_{I Q 4}\right)$ respecto a los cálculos teóricos. Los errores porcentuales de las simulaciones respecto a los cálculos para cada dispositivo $x$ pueden obtenerse mediante $\varepsilon_{P x s i m}=\left(P_{x s i m}-P_{x c a l c}\right) / P_{x c a l c}$. Asimismo se pueden obtener los errores porcentuales para los datos experimentales: $\varepsilon_{P x e x p}=\left(P_{x e x p}-P_{x c a l c}\right) / P_{x c a l c}$.

Tabla 1: Potencias disipadas en los dispositivos dadas en vatios en función de $M$

\begin{tabular}{|c|c|c|c|c|c|c|c|c|c|}
\hline$M$ & 0,2 & 0,3 & 0,4 & 0,5 & 0,6 & 0,7 & 0,8 & 0,9 & 1 \\
\hline$P_{I Q 1}$ (calculada) & 0,01089 & 0,04620 & 0,10558 & 0,18912 & 0,29617 & 0,42657 & 0,58053 & 0,75716 & 0,95678 \\
\hline$P_{I Q 1}($ simulada $)$ & 0,02925 & 0,07841 & 0,14717 & 0,23674 & 0,34632 & 0,47741 & 0,63029 & 0,80304 & 0,99841 \\
\hline$P_{I Q 1}$ (medida) & 06429 & 0,12888 & 0,22790 & 0,35865 & 0,49988 & 0,67316 & 0,84579 & 1,02590 & 1,19593 \\
\hline$P_{I Q 4}$ (calculada) & 0,16924 & 0,35802 & 0,54422 & 0,72941 & 0,91169 & 1,09168 & 1,27046 & 1,44644 & 1,62084 \\
\hline$P_{I Q 4}($ simulada $)$ & 0,16117 & 0,34747 & 0,53187 & 0,71495 & 0,89848 & 1,08052 & 1,26122 & 1,44119 & 1,61900 \\
\hline$P_{I Q 4}($ medida $)$ & 0,38560 & 0,58796 & 0,79150 & 1,00920 & 1,20273 & 1,40522 & 1,57035 & 1,68868 & 1,87298 \\
\hline$\varepsilon_{P I Q 4 \exp }(\%)$ & 127,84 & 64,23 & 45,44 & 38,36 & 31,92 & 28,72 & 23,60 & 16,75 & 15,56 \\
\hline$P_{D 4}($ calculada $)$ & 0,15875 & 0,31350 & 0,44247 & 0,54715 & 0,62625 & 0,68056 & 0,71095 & 0,71671 & 0,69872 \\
\hline$P_{D 4}($ simulada $)$ & 0,13039 & 0,26781 & 0,38368 & 0,47858 & 0,55349 & 0,60727 & 0,63996 & 0,65170 & 0,64253 \\
\hline$P_{D 4}($ medida $)$ & 0,27852 & 0,41125 & 0,50865 & 0,59505 & 0,63808 & 0,63940 & 0,61826 & 0,57743 & 0,52554 \\
\hline$P_{D 1}($ calculada $)$ & 0,00039 & 0,00167 & 0,00382 & 0,00685 & 0,01073 & 0,01545 & 0,02103 & 0,02743 & 0,03466 \\
\hline$P_{D 1}($ simulada $)$ & 0 & 0 & 0 & 0 & 0 & 0 & 0 & 0 & 0 \\
\hline$P_{D 1}($ medida $)$ & 0,00056 & 0,00055 & 0,00124 & 0,0024 & 0,00321 & 0,0015 & 0,0002 & 0,00057 & 0,00952 \\
\hline
\end{tabular}

De acuerdo con los resultados de la figura 6, puede decirse que las deducciones teóricas son acordes con los resultados experimentales. En las tres figuras puede notarse la misma tendencia en las curvas experimentales, simuladas y calculadas. Sin embargo los datos presentan un error porcentual de acuerdo con los cálculos. Estos errores están relacionados con los bajos valores del índice de modulación $(M)$, a medida que crece $\mathrm{M}$ el error entre las mediciones y las predicciones teóricas disminuyen. Al referirse a las figuras 2 y 3 se puede observar que para índices de modulación pequeños los pulsos sobre G4 durante los extremos del semiciclo (cercanos a 0 y $\pi$ ) aumentan su duración y disminuyen su distancia relativa. Estas distancias pueden perderse para una gran cantidad de pulsos, es decir, para altas frecuencias de conmutación. 


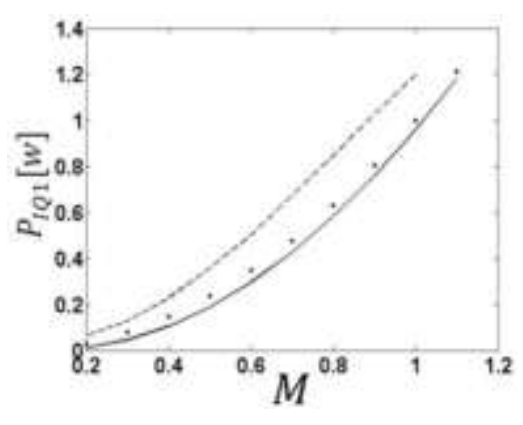

a)

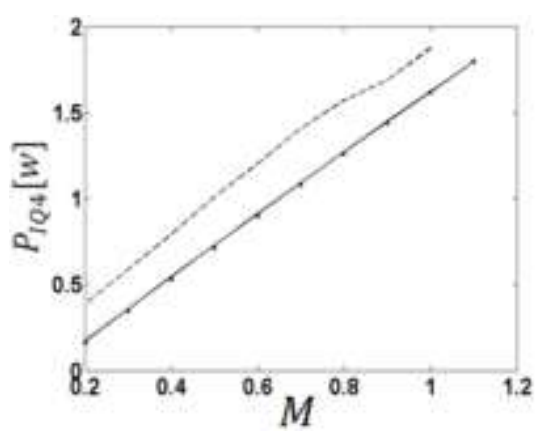

b)

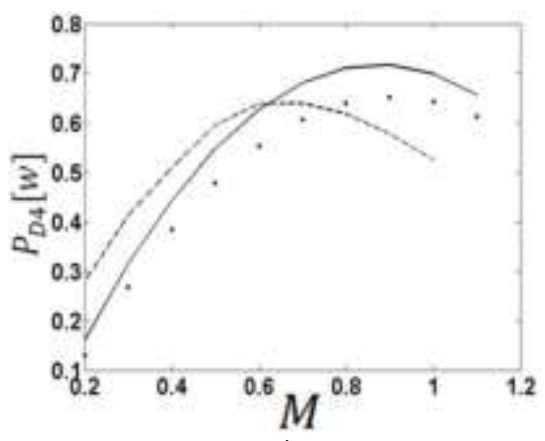

c)

Fig. 6: Pérdidas de potencia en vatios calculadas (-), simuladas (.) y experimentales (--) en función del índice de modulación a) IGBT1. b) IGBT 4. c) diodo 4.

Los microcontroladores tienen limitantes en la resolución de los pulsos de PWM que envían hacia los IGBT. Además de esto, los IGBT no responden de forma inmediata a los cambios de estado en tiempos cortos. Estos factores afectan las propiedades geométricas de la modulación que se consideran en los análisis realizados. De igual manera se ha llevado a cabo una medida indirecta para medir las pérdidas en cada dispositivo con ayuda de la ecuación (20) que introduce errores asociados a la adquisición de los datos. Tampoco hemos considerado las variaciones en la caída de voltaje en los semiconductores, los transitorios no discretos de los interruptores, entre otros.

Remitiéndose a la figura 3a, en relación con la potencia disipada en Q1, se observa que a medida que crece $M$ crecen tanto el tiempo de conducción de Q1 como su corriente (al ser ésta proporcional a $M$ ). Es decir, la potencia disipada en Q1 depende de estos dos factores diferenciables que se incrementan en la medida en que se incrementa $M$. La ecuación (17) logra un gran acercamiento a este fenómeno. En relación con la potencia disipada en Q4, se observa de la figura $3 a$ que existen dos intervalos de conducción: $(0, \theta)$ y $(\pi+\theta, 2 \pi)$. El intervalo $(0, \theta)$ es más corto que el segundo para las condiciones específicas de validación. Con base en esta premisa se encuentra que el intervalo $(\pi+\theta, 2 \pi)$ de Q4 influye más fuertemente para el cálculo de la potencia disipada. En el intervalo $(\pi+\theta, 2 \pi)$ solo el aumento de la corriente influye en el aumento de la potencia disipada. Lo que lleva a una relación lineal entre esta potencia y el índice de modulación expresadas en (18).

Para la potencia disipada en D4 se observa de la figura 3a que hay dos intervalos de conducción: $(\theta, \pi)$ y $(\pi, \pi+$ $\theta$ ). En el intervalo $(\theta, \pi)$ disminuye el tiempo de conducción debido a que disminuye el índice de modulación y además disminuye la corriente debido a la relación lineal con $M$. Esto lleva a que la potencia disipada en D4 durante este intervalo disminuya con el cuadrado de $M$. En el intervalo $(\pi, \pi+\theta)$ la potencia depende sólo de la corriente ya que la conducción es continua. Al superponer las respuestas de ambos intervalos se llega a la suma de una cuadrática negativa y de una recta de pendiente positiva tal como está expresado en la ecuación (19).

\section{CONCLUSIONES}

El artículo presenta un análisis de las pérdidas de potencia en semiconductores de un inversor SPWM que incluye cálculos teóricos, simulaciones y experimentos:

1) Las ecuaciones (17), (18) y (19) describen concretamente el comportamiento de la potencia en los dispositivos bajo las condiciones de la prueba. Estas ecuaciones son aproximaciones validas de las ecuaciones (9), (12) y (15) dadas las condiciones experimentales bajo las que se analizaron las potencias y su comportamiento en función del índice de modulación $M$. La premisa de que estas ecuaciones siguen el comportamiento descrito en la figura 6 con $K_{1}, K_{2}, K_{3}$ y $K_{4}$ como constantes es una aproximación racional que muestra la validez de las ecuaciones y linealidad en los rangos de medición descritos.

2) Las relaciones geométricas de la modulación han permitido expresar las pérdidas por conducción en ecuaciones simplificadas que sólo dependen de $M$ para una carga $\mathrm{RL}$ específica. Esto permite el análisis y la evaluación de las pérdidas por conducción para inversores y puede ser base para estudios futuros permitiendo modelar la eficiencia de los inversores. 
3) Las pérdidas de potencia en los semiconductores tienen una fuerte dependencia de los parámetros de la modulación SPWM. Las ecuaciones obtenidas describen muy fielmente el comportamiento de la potencia disipada. Sin embargo un estudio posterior consistiría en evaluar las pérdidas de potencia como función del ángulo del factor de potencia $\theta$ y con base en la obtención de factores empíricos que ajusten mejor las curvas experimentales con las teóricas.

4) el análisis de las formas de onda en los interruptores permite visualizar la semejanza de las ecuaciones (17), (18) y (19) con las variaciones en la corriente a través de ellos y el índice de modulación. El comportamiento de la corriente y su relación con el índice de modulación, y por tanto, con los tiempos de conducción de los interruptores puede seguirse gráficamente a través de la figura 3. Observando las formas de onda de la figura 3 , el fenómeno descrito de manera abstracta mediante estas ecuaciones se ve concretamente al variar el índice de modulación.

\section{AGRADECIMIENTOS}

Los autores agradecen a la Universidad de Antioquia (UdeA) por el apoyo de "Sostenibilidad 2013-2014". Igualmente se agradece al CODI de la UdeA por financiar el proyecto "Diseño y construcción de Prototipo Convertidor Trifásico DC-AC".

\section{REFERENCIAS}

Bazzi, A.M.,Krein, P.T., Kimball, J.W., yKepley, K.,IGBT and Diode Loss Estimation Under Hysteresis Switching. IEEE Trans. Power Electron, 27(3), 1044-1048 (2012)

Bierhoff, M.H., y Fuchs, F.W., Semiconductor losses in voltage source and current source IGBT converters based on analytical derivation, In Power Electronics Specialists Conference 2004 IEEE 35th Annual, 4(1), 28362842(2004)

Casanellas, F., Losses in PWM inverters using IGBTs, Electr. Power Appl. IEE Proc., 141(5), 235-239, (1994)

Dahono, P.A., Sato, Y., y Kataoka, T., Analysis of conduction losses in inverters, Electr. Power Appl. IEE Proc., 142(1), 225-232, (1995).

Gallego, J., Muñoz, N., Cano, J.,Análisis de Flujos de Potencias Ineficientes Producidos por Convertidores de Potencia, Información Tecnológica 25(3), ON PRESS (2014)

Hosseini Aghdam, M.G., y Gharehpetian, G.B., Modeling of switching and conduction losses in three-phase SPWM VSC using switching function concept, In Power Tech, 2005 IEEE Russia, 1(1), 1-6, (2005)

Ismail, B., Taib, S., Saad, A.R.M., Isa, M., y Hadzer, C.M., Development of a Single Phase SPWM Microcontroller-Based Inverter. In Power and Energy Conference, 2006. PECon '06. IEEE International, 1(1), 437-440, (2006)

Jacobina, C.B., dos Santos, E.C., Rocha, N., y Lopes Fabricio, E.L., Single-Phase to Three-Phase Drive System Using Two Parallel Single-Phase Rectifiers, IEEE Trans. Power Electron, 25(5), 1285-1295, (2010)

Kojabadi, H.M., A comparative analysis of different pulse width modulation methods for low cost induction motor drives, Energy Convers. Manag., 52(1), 136-146, (2011)

Komurcugil, H., Rotating-Sliding-Line-Based Sliding-Mode Control for Single-Phase UPS Inverters, IEEE Trans. Ind. Electron, 59(10), 3719-3726, (2012)

M. N. Md Isa, M.I.A.,FPGA Based SPWM Bridge Inverter. American Journal of Applied Sciences, 4 (8),584-586, (2007)

Perantzakis, G.S., Xepapas, F.H., y Manias, S.N., A Novel Four-Level Voltage Source Inverter mdash;Influence of Switching Strategies on the Distribution of Power Losses. IEEE Trans. Power Electron, 22(1), 149-159, (2007)

Rus, D.C., Preda, N.S., Incze, I.I., Imecs, M., y Szabó, C., Comparative analysis of PWM techniques: Simulation and DSP implementation, IEEE International Conference on Automation Quality and Testing Robotics (AQTR), $3(1), 1-6,(2010)$ 
Trigg, M.C., Dehbonei, H., y Nayar, C.V., Digital sinusoidal PWMs for a micro-controller based single-phase inverter, Part 1:Principles of digital sinusoidal PWM generation. Int. J. Electron., 95(8), 819-840, (2008)

Wang, Z., Fan, S., Zheng, Y., y Cheng, M.,Design and Analysis of a CHB Converter Based PV-Battery Hybrid System for Better Electromagnetic Compatibility. IEEE Trans. Magn.,48(11), 4530-4533, (2012)

Xiao, C., Chen, G., y Odendaal, W.G., Overview of power loss measurement techniques in power electronics systems. In Industry Applications Conference, 2002. 37th IAS Annual Meeting. Conference Record of the, 2(1), 1352-1359, (2002)

Xiao, H., Xie, S., Chen, Y., y Huang, R.,An Optimized Transformerless Photovoltaic Grid-Connected Inverter. IEEE Trans. Ind. Electron. 58(5) , 1887-1895, (2011) 